\title{
HYBRID MAC PROTOCOL FOR WIRELESS SENSOR NETWORKS USED IN TIME CRITICAL APPLICATIONS
}

\author{
Pandeeswaran Chelliah $^{1}$, Pappa Natarajan ${ }^{2}$, and Jayesh Sundar Gopinath ${ }^{3}$ \\ ${ }^{1}$ Research Scholar, Dept of Instrumentation Engineering, MIT Campus, \\ Anna University, Chennai- 44 \\ cpandees @gmail.com \\ ${ }^{2}$ Professor, Dept of Instrumentation Engineering, \\ MIT Campus, Anna University, Chennai- 44 \\ npappaerediffmail.com \\ ${ }^{3}$ PG Scholar, Dept. of Electronics and Instrumentation, \\ St.Joseph's College Of Engineering \\ gjayeshsundar91@gmail.com
}

\begin{abstract}
In this paper a H-MAC protocol (Hybrid Medium Access Control protocol) has been proposed, which is an energy efficient and low latency MAC protocol which uses node ID method to assign priority for certain wireless sensor nodes that are assumed to be present in critical loops for an industrial process control domain. H-MAC overcomes some of the limitations in the existing approaches. In the case of industrial automation scenario, certain sensor loops are found to be time critical, where data's have to be transferred without any further delay, as failure in immediate transmission leads to catastrophic results for humans as well as machinery in industrial domain. The proposed H-MAC protocol is simulated in NS2 environment, from the result it is observed that the proposed protocol provides better performance compared to the conventional MAC protocols mentioned in the recent literature for the conceded problem.

A MAC protocol which provides both energy saving mechanism and that can handle emergency situation is the most desired for any industry. In any industry time and mission critical scenarios requires strict timeliness and reliability along with the energy efficiency. However there are dynamic and harsh environmental conditions for which the MAC protocol must survive and do transmission accordingly. The dynamic changes in topology must also be adapted so that the nodes are in constant link to the destination. Most of the existing MAC protocols have been identified as they face a number of limitations for industrial application domain.
\end{abstract}

\section{KEYWORDS}

MAC protocol, Industrial wireless sensor networks (IWSN), Time critical applications and Energy efficiency.

\section{INTRODUCTION}

Research of the past years has led to numerous novel development and approaches for wireless sensor networks. Energy efficiency is a critical issue for sensor networks, where nodes work with resource constraint battery power. Recent advancement in wireless communication and device technology has enabled the development of low cost sensor networks composed of tiny sensors.

Dhinaharan Nagamalai et al. (Eds) : ACITY, WiMoN, CSIA, AIAA, DPPR, NECO, InWeS - 2014 pp. 125-135, 2014. (C) CS \& IT-CSCP 2014

DOI : $10.5121 /$ csit.2014.4514 
The sensor nodes are typically capable of sensing, processing, and networking. Since a sensor node is a small, lightweight, un-tethered, battery-powered device, its energy is limited [1], [2], [3]. As a result, energy efficiency is a critical issue for sensor networks. Many researchers have focused on the development of power saving schemes for wireless sensor networks [10], [11], [12], [13]. These schemes include power saving hardware and topology design, power-efficient MAC (medium access control) layer protocol/ network layer routing protocol. Even though the research field of wireless sensor networks and in particular the MAC protocols is relatively new, there exist numerous MAC protocols proposed in the recent literature, designed specifically for wireless sensor networks.

Nowadays WSN have been extended to support many application domains such as military target tracking, industrial automation or patient monitoring. The aforementioned conventional MAC protocol is no longer adequate for these application domains. For example sensor observing pressure in pipes must deliver messages to an actuator connected to a valve in a timely and reliable fashion. Another example in power plant boiler process control, apart from other parameters, pressure and level must be controlled in timely and reliable fashion. Hence, to support time critical and mission critical applications a necessary first step is to find a MAC protocol that is capable of supporting performance bounds on data transport delay and reliability.

A large number of MAC protocols for wireless sensor networks have been proposed in literatures [10], [11], [12], [13]. It is believed that the data transport delay and reliability are two important objectives most relevant in the context of mission critical and time critical applications, while energy efficiency could be addressed additionally if required.

Communication in wireless sensor networks can be divided into several layers like other communication infrastructure. The MAC layer, which is primarily responsible for providing accessibility to the channel for communication. It is described by a MAC protocol, which tries to ensure that no two nodes interfere with each other during communication using a proper coordination mechanism. In general, the main design goal of typical MAC protocols is to provide high throughput, minimized latency, fairness, and quality of service. In addition, the MAC protocol for wireless sensor network needs to consider energy efficiency because of the limited energy of constituent sensor nodes. The primary design issue for the MAC protocol of wireless sensor network is thus, how to support the basic functions of MAC protocol while minimizing energy consumption of the sensor nodes to maximize the lifespan of the network.

In Bluetooth or 802.11 , Energy conservation is not a primary objective, because mostly nodes are charged every day or mains powered. The commercial standards like IEEE 802.11 define a power management scheme for ad hoc networks, wherein the nodes remain in idle listening state to conserve the energy in low traffic condition. It was shown that a significant amount of energy can be wasted even in the idle listening mode.

Hence, IEEE 802.11 is not suitable for sensor networks. S-MAC is a MAC protocol designed specifically for wireless sensor networks. It forces the sensor nodes to operate with low duty cycle and take periodic sleep instead of idle listening. The sensor nodes also sleep during overhearing period to save the energy [2], [3]. Although S-MAC can save more energy than IEEE 802.11 protocol, it cannot efficiently adapt to the network traffic condition since it uses a fixed duty cycle for all the sensor nodes. A duty cycle tuned for high traffic loads results in a waste of energy when the traffic is low, while tuning for low traffic loads results in low throughput under high traffic loads. The Timeout-MAC protocol (T-MAC) improves the S-MAC protocol by employing the approach of adaptive duty cycle. If there is no activity in the vicinity of a node for a while, it sleeps. Such an adaptation frees the application from the burden of selecting an appropriate duty cycle. T-MAC displays the same performance as S-MAC under constant traffic loads, but saves more energy under variable traffic [4]. 
In this paper a H-MAC protocol proposed, which determines end to end delay and adaptively determines the transmission schedule according to the buffer condition and the context of the packets. The existing approaches designed for wireless sensor network improve energy efficiency by controlling the duty cycle. The proposed protocol reduces energy consumption by letting each node stay in the sleep mode if the number of packets in the buffer is smaller than the threshold, while the threshold value is decided according to the distance of the node to the sink node. The variable threshold for each switch node may cause increased latency, Since certain sensor loops in industries are found to be of critical loops data's have to be transferred without any further delay, thus the contention access period for these prioritized nodes can be decided if the sensed information is of time critical, and transferring the data immediately to the sink.

The rest of the paper is presented as follows. Section 2 reviews the related work. Section 3 presents the proposed approach that use H-MAC protocol. Section 4 reveals about results acquired and discussion. Finally section 5 concludes the paper and outlines the future research direction.

\section{RELATED WORK}

Due to the energy constrained environment, the MAC protocol for sensor networks has to take energy efficiency as one of its primary concerns. The existing wireless MAC protocols such as Bluetooth and 802.11 MAC protocols [6] cannot be directly applied to the sensor networks since none of them take energy conservation as the primary design objective.

There have been several MAC protocols specially designed for sensor networks. S-MAC is a MAC protocol with periodic listen/sleep scheduling based on local synchronization. In the SMAC protocol, the listen and sleep period are set to be a fixed length. During the listen period, SYNC and RTS/CTS control packets are transmitted based on the CSMA/CA mechanism for the purpose of synchronization and announcement of the succeeding data packet transmission. Any two nodes exchanging the RTS (Request-to-Send)/CTS (Clear-to-Send) packet in the listen period stays in the wake state and start data transmission during the sleep period of other nodes. All other nodes can enter the sleep mode to conserve the energy. Generally, periodic listen/sleep has the trade-offs between energy saving and latency. To improve the performance, S-MAC uses an adaptive listening scheme in which the node receiving NAV information remains awake and tries to communicate in the sleep mode without waiting for the next listen/sleep cycle. In order to decrease the latency of S-MAC, DSMAC [5] supports multiple duty cycles automatically adjusted according to the energy consumption level and delay. T-MAC improves the energy efficiency of S-MAC by using a very short listening window at the beginning of each active period. The length of active time is adaptive, and the timer is defined by equation (1)[4].

$$
\mathrm{T}_{\text {out }}=\mathrm{C}+\mathrm{R}+\mathrm{T}
$$

$\mathrm{C}$ is the contention interval, $\mathrm{R}$ is the length of RTS packet, and T is a very short time interval between RTS and CTS that is identical to SIFS (Short Inter Frame Space) in 802.11 MAC. If no data is transmitted during Timeout, the active nodes enter the sleep mode for saving the energy until the beginning of the next listening period. If no activity occurs in that period, the node returns to the sleep mode by adapting the duty cycle. T-MAC thus saves the energy at a cost of reduced throughput and increased latency. With the same workloads, T-MAC and S-MAC perform equally, while T-MAC suffers from the same complexity and scaling problem of SMAC. Shortening the active window in T -MAC reduces the ability to snoop on the surrounding traffic and adapt to the changing network condition. 


\subsection{Unique Requirements of Industrial WSN}

At the Media Access Control (MAC) layer Energy efficiency becomes primary concern in designing MAC protocol to maximize network lifetime. Deterministic MAC layer design required to achieve low latency and reliable delivery of messages to the destination.ISA SP 100 Working group classified industrial process control in to six different classes based on latency

Class 0: Emergency action (in terms of micro seconds)

Class 1: Closed-loop regulatory control (in terms of milliseconds)

Class 2: Closed-loop supervisory control (in terms of seconds)

Class 3: Open-loop control (in terms of minutes)

Class 4: Monitoring with short-term operational consequences (in terms of hours)

Class 5: Monitoring without immediate operational consequences. (in terms of days)

\section{THE PRIORITIZING APPROACH}

In order to satisfy the unique requirements of sensor and control devices a suitable MAC protocol must be devised. In the proposed prioritizing approach, it is planned to give priority only to sensor nodes which are in the critical loop during contention access period of super frame structure, more importantly collision avoided by giving channel access to nodes in critical loop. Reduced collisions and transmissions in turn will consequently reduce power consumption. Worst case delay for the urgent packets is the one cycle time. The contexts collected by the sensors are diverse because of the inherent characteristics of wireless sensor network. Also, the types and importance of the contexts are all different. There might be some context data requiring urgent transmission, while the urgency varies according to the location of the sensors. On the other hand, there are some sensors located in such places requiring little monitoring. We use the new approach deciding the operation of the nodes based on the contexts.

H-MAC protocol changes the state of a node according to the quantity of accumulated data in the buffer and the importance of the context before the contention period begins.

\subsection{Proposed Approach}

The basic mechanism involved in this Hybrid MAC PROTOCOL involves a series of steps after deploying the nodes and they are as follows,

Step 1- The protocol identifies the near one hop neighbors by broadcasting the ping message from its location. The ping message informs the network nodes begin by first identifying the one hop neighbors from its location. This is feasible by broadcasting ping message once in a while. The ping message exchanges neighbor's location to each other and also to inform the sleep and wake cycles. This is done every 30 seconds in this simulation [4]. And the ping memory is then exchanged between nodes, thus enabling the nodes to have two hop neighbors list.

Step 2- Initially CSMA mechanism is followed as the traffic will be low and thus data transfer occurs by sensing the carrier. However the traffic is never the same and as the traffic increases beyond the threshold the CSMA mechanism is dropped.

Step 3- The node that is present in a high priority loop must be given first priority, thus using the node ID the current transmission is dropped and changes over to TDMA thereby giving the first slot to the node that is in the high priority loop.

Step 4- If two nodes that are present in the same high priority region, then slots are assigned to the nodes one after the other. And after the transmission the network changes back to CSMA. 
Step 5-The fast transmission using the node id is done only for critical loops, this node id information is passed along the header packet before the data packets being sent.

However, this approach does not considers the importance of important nodes getting access of channel in case of emergency or time critical situation, because in many industries most nodes are powered by wire and thus energy efficiency is not the only constraint. A time critical information have to be transferred quicker than any other data, thus the best approach is by getting a hold of the channel, that is the contention access period of the node should be flexible in case of time critical information. This feature is deployed to certain nodes only, i.e. nodes that are present in critical loops in and around the industry. Thus the data is transferred to the sink quicker as the channel is being taken over for quicker transfer.

\subsection{A Decision of Threshold Value}

In the H-MAC protocol the packets are transmitted only when the height of the buffer exceeds the specified threshold value. This can assure the energy efficiency. Each node switches to the sleep mode if the number of packets in the buffer is smaller than the specified threshold value or an RTS packet has not been received when the timer is on. To reduce unnecessary idle listening time after data transmission, the proposed H-MAC lets the node switch to the sleep mode and conserve the energy when there is no data to receive. This will substantially reduce the energy consumption since a node has more chances of sleep. However, if all nodes have the same threshold value, the effectiveness will be valid in only the single-hop network. For example, assume that a node is waiting for transmission until its buffer exceeds the specified threshold value. As soon as the buffer reaches the threshold value, the node transmits the packets to the next node. However, the buffer of the next node will be full as soon as it receives them since they have the same buffer threshold.

The figure shown in the figure 2 explains an industrial scenario that is filled with both high priority and low or normal priority loops. The network consists of several end devices and routers that transfer the data from one loop to the other towards the sink. There is a single central co coordinator node that governs the network protocols and proper time slotting for all the nodes. The nodes in high priority loops are the ones that are given the top priority in transferring the data; the node ID as mentioned in the algorithm enables this feature. The router holds the routing table in order to establish route on demand.

Therefore, the protocol decides the threshold value of a node according to the hop-count from the sink node to maximize the energy efficiency and also to apply to the multi-hop networks. Fig. 2 shows that each node has a different threshold value according to the hop-count from the sink in the proposed H-MAC protocol. If node-D transmits a packet to node-

$\mathrm{C}$ after its buffer exceeds the threshold value, node-C is able to get a chance of sleep since its threshold value is bigger than the preceding node, node- $\mathrm{D}$. As a result, each node can improve the energy efficiency. We use two parameters, $\alpha$ and $\lambda$, to decide the threshold value, where $\alpha$ is the parameter reflecting the hop count while $\lambda$ is the degree of the change of threshold value. When $\alpha$ is bigger than or equal to the difference between the hop-count of the source packet(Ntotal) and the hop count to the sink( Nown), the threshold value, Qthresh, is determined by Equation-(2) below the figure 2. Both these equations are used in the network based upon the nodes position. 


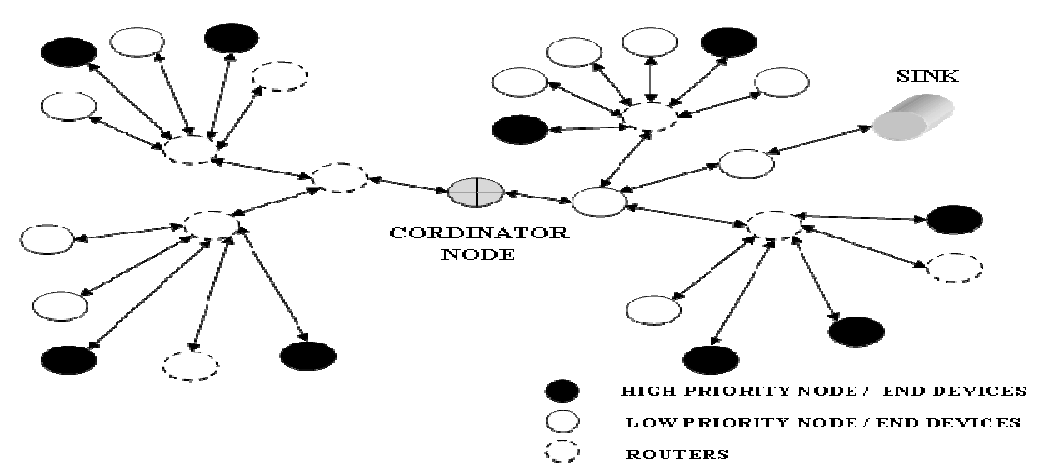

Fig 1. Network Scenarios in Industries.

$$
\begin{array}{ll}
Q_{\text {threshold }}=[(\lambda-\alpha) 10] & \alpha<\lambda \\
Q_{\text {threshold }}=[(\alpha) 10] & \alpha=\lambda
\end{array}
$$

The existing approach uses greedy based algorithm for routing, which has a major setback of not proceeding in the most optimal way, whereas here we use an optimal link state routing which improves the network performance than the existing approach.

The first equation is considered for nodes that are except for the last node in the network towards the sink. And the second equation is used for the node that was left in the first. Thus we save energy by the varying buffer level.

In the above two equations, $\alpha$ is the current node in the gird, while $\lambda$ is the total number of nodes in the sensor network. If there are 10 nodes then $\lambda$ is 10 , then the 10th node or first node from sink then it will have 100 has its threshold level. If current node $\alpha$ is 8 or the third node from sink, then $Q_{\text {threshold }}$ for $\lambda$ of 10 will be 80 .

This feature can be easily visualized for grid topology, however in the case of random since any nodes can be in the path of network the data's are being transferred via the shortest possible route to the destination. In the case of an emergency data the priority is authorized to that particular nodes information. The following section explains the result of the proposed hybrid MAC protocol.

\section{RESULTS AND DISCUSSIONS}

The simulation is performed using network simulator 2 for two different scenarios, they are grid and random topology. The grid topology is that the nodes are connected together in a matrix form and thus named as grid topology. The random topology has no predefined order or manner for the nodes, and thus they are deployed in random locations. The proposed protocol is compared with the some of the existing protocols such as S MAC T MAC. The Figures explains energy consumed by nodes linearly increases for all the protocols with respect to number of nodes. The grid topology has a fixed distance between the nodes and the network size increases as the number of nodes increases. Gradually the number of nodes increased from 16 to 100 . For any IWSN the two factors that play the key roles are reliability and timeliness, to satisfy this real time link state routing protocol is used in this approach which ensures reliability and timeliness.

H-MAC consumes significantly lesser energy; this is done by reducing and setting threshold value for deciding the transmission. Lower power consumption is also achieved avoiding CAP collision by giving the bandwidth access to the nodes on high priority nodes. Fig 3(b)illustrate the 
control packet overhead, specifically the number of RTS, CTS and ACK packets used by H-MAC with that of SMAC and TMAC. It is very clear from the graphs that the total number of packet overhead is significantly reduced. It is also noted that transmission of packets are also reduced by transmitting only when the specified threshold value is exceeded, thus conserving energy in every possible way. This mechanism greatly reduces the control packet overhead.

The latency in the case of H MAC for normal transmission is found to be the most delayed transmission when compared to that if the existing, however this is done to conserve energy as much as possible. On the other hand there are time critical data's and for important packets that are marked as H-MAC, the latency is much lower than SMAC and TMAC protocols, which is another critical requirement of IWSN. The latency of H-MAC for important packets is close to that of MAC with no sleep, since the data packets of important context are allowed to be immediately transmitted to the next node.

\subsection{PERFORMANCE ANALYSIS}

The performance analysis is evaluated using the graph obtained from the simulation. The values are the ones that are present at the end of simulation. The simulation lasts for $120 \mathrm{~ms}$ and the below values are adapted from the respective graphs.

TABLE 1: PACKET DELIVERY RATIO

\begin{tabular}{|c|c|c|}
\hline \multirow{2}{*}{ MAC } & \multicolumn{2}{|c|}{ Packet Delivery Ratio } \\
\cline { 2 - 3 } & $\begin{array}{c}\text { Grid } \\
\text { Topology }\end{array}$ & Random Topology \\
\hline H MAC & 0.9620 & 0.9600 \\
\hline Z MAC & 0.9570 & 0.9475 \\
\hline T MAC & 0.9560 & 0.9450 \\
\hline S MAC & 0.9525 & 0.9400 \\
\hline
\end{tabular}

TABLE 2: AVERAGE END TO END DELAY

\begin{tabular}{|c|c|c|}
\hline \multirow{2}{*}{ MAC } & \multicolumn{2}{|c|}{ Average End to End Delay(ms) } \\
\cline { 2 - 3 } & $\begin{array}{c}\text { Grid } \\
\text { Topology }\end{array}$ & Random Topology \\
\hline H MAC & 42 & 44 \\
\hline Z MAC & 50 & 54 \\
\hline T MAC & 84 & 86 \\
\hline S MAC & 76 & 77 \\
\hline
\end{tabular}

\begin{tabular}{|c|c|c|}
\hline \multirow{2}{*}{ MAC } & \multicolumn{2}{|c|}{ Residual Energy(Joules) } \\
\cline { 2 - 3 } & Grid Topology & Random Topology \\
\hline H MAC & 71 & 71 \\
\hline Z MAC & 69 & 67 \\
\hline T MAC & 67 & 65 \\
\hline S MAC & 63 & 63 \\
\hline
\end{tabular}

TABLE 3: RESIDUAL ENERGY 


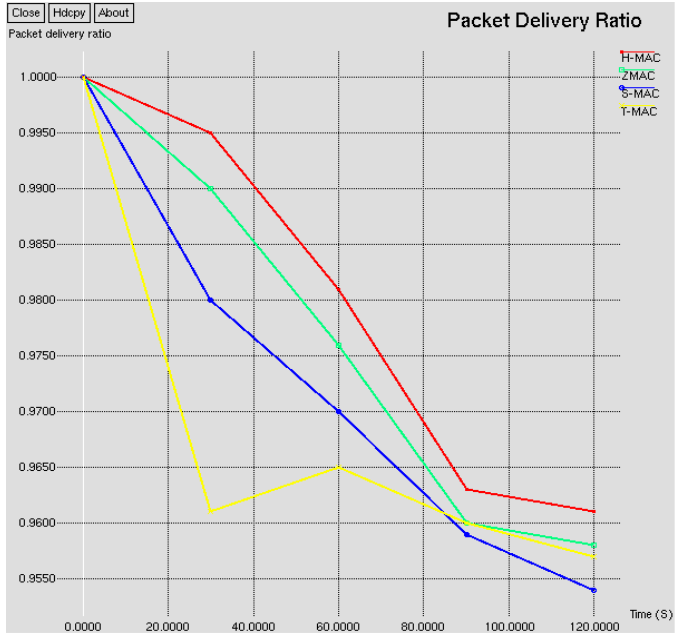

Fig 2. Packet Delivery Ratio (grid)

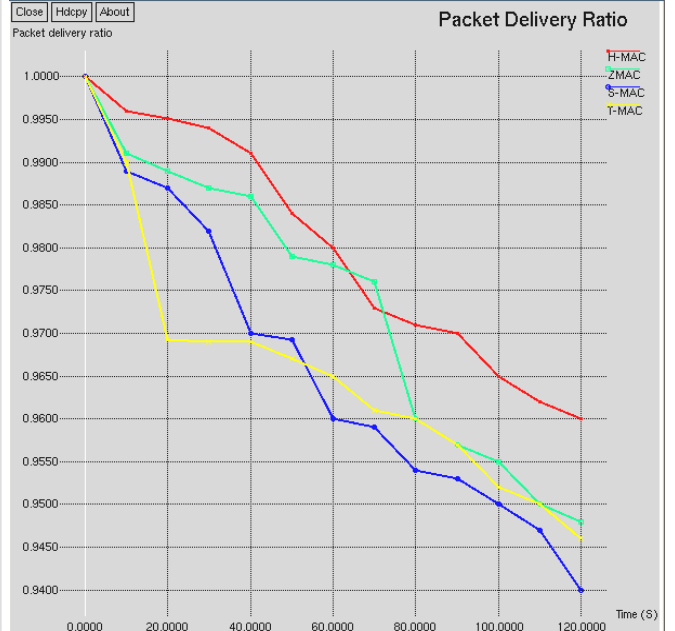

Fig 3. Packet Delivery Ratio (random)

Packet Delivery Ratio-The packet delivery ratio in general is the ratio between "the total numbers of packets sent from the source to that of the total number of packets received in the destination". This ratio indicates the successful ability of the protocol developed in any scenario as the ultimate aim is to transfer data.

From the graph analysis it can be clearly understood that the H MAC are being capable of separating the high priority information and transfer the data to the destination. The comparison clearly indicates that the proposed H MAC on comparison with the existing protocol is much better and has a marginal difference to that of the existing protocol as seen in the graph. The simulation shows different colour for different protocols, the simulation time is for 120 seconds with 100 nodes at any instant.

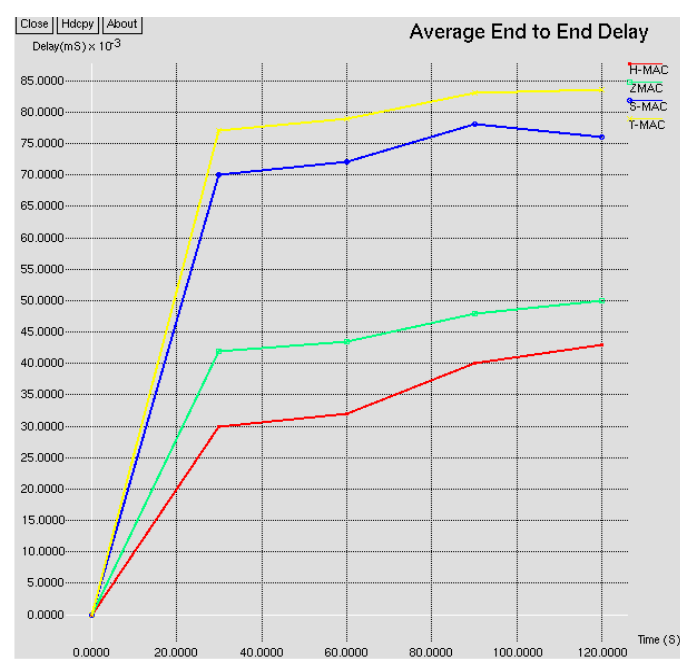

Fig 4. Average End to End delay (grid)

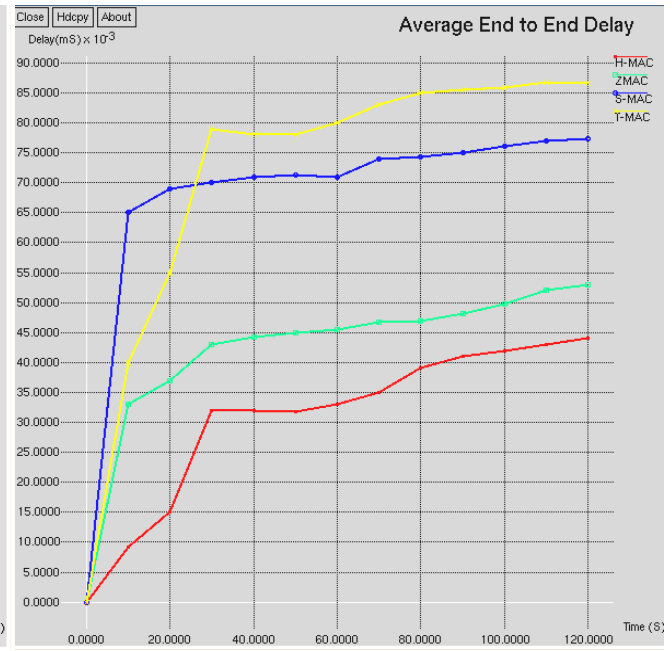

Fig 5. Average End to End delay (random)

Average End to End Delay- The delay that occurs as the data travels through the network from the source to the destination is the end to end delay. It is clearly observed that the HAC has the least end to end delay of $\sim 43$ (approx.). The major advantage in the hybrid MAC protocol is 
that information here is immediately transferred however in the existing protocols had to wait and transfer data only in the next wake cycle or only by informing the other nodes in prior. In the case of $\mathrm{H}$ MAC the delay is greatly reduced with the aid of the buffer memory and prioritizing nodes which enables quick and safe delivery of information.

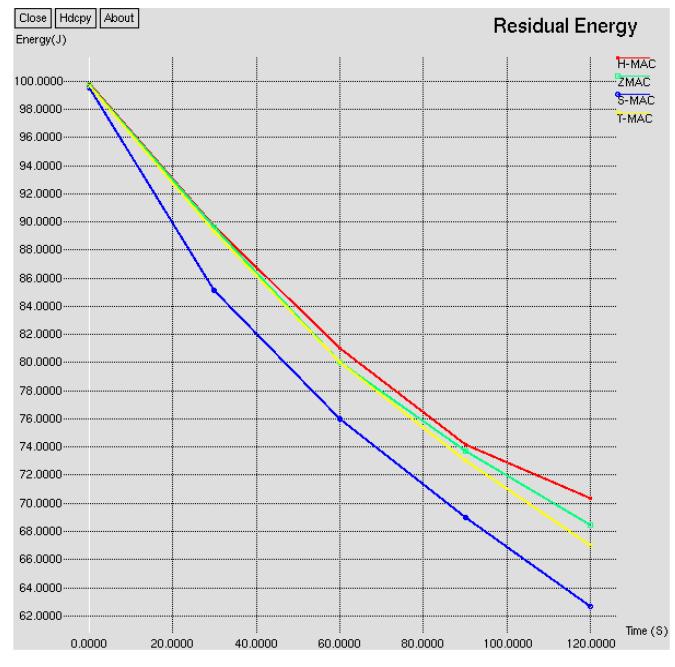

Fig 6. Residual Energy (grid)

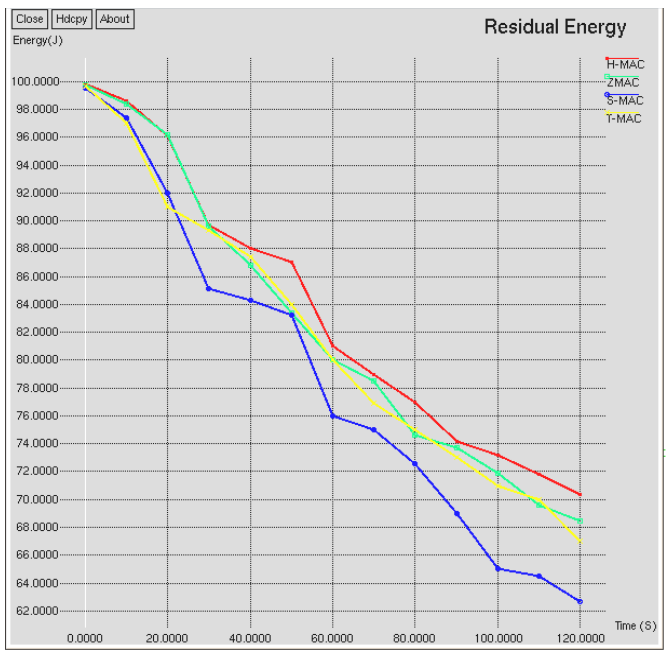

Fig 7. Residual Energy (random)

Residual energy- The energy left in any node at any instant is said to be the residual energy. The initial energy of each node is 100 joules. The transmission, route discovery, priority checking, and reception are some of the major reasons for energy loss in a network, it is thus found that this protocol is efficient as it consumes lesser energy.

From the graph it is clearly observed that the S MAC with a $50 \%$ duty cycle consumes the most energy while H MAC protocol consumes the least amount with the aid of the changing buffer level in each node, which reduces the energy consumption.

\section{CONCLUSION AND FUTURE WORK}

The Carrier sense medium access provides the network to transfer data in low traffic scenario to conserve energy; however the time division medium access have the ability to transfer in high traffic scenario there by transferring data's as efficient as possible.

The proposed protocol with the above features together enables it to outperform the existing protocol. The reason why H MAC outperforms when compared to that of the Z MAC is that its feature of changing buffer (memory) level. The reliability of the path may be improved by link state routing and end to end delay may be further reduced by using cross layer approach, thereby saving more energy.

However the protocol has to be advanced by reducing the time consumption in changing over from TDMA to CSMA. In order to further reduce the energy consumption variable memory level has to be set to change from that instant, based upon the distance from that node to the sink, which will enable the nodes anywhere in the network to save energy. 


\section{REFERENCES}

[1] Akyildiz, W. Su, Y. Sankarasubramaniam and E. Cayirci, A survey on sensor networks. IEEE Communications Magazine, 40(8), pages 102-114, 2002.

[2] G W. Ye, J. Heidemann, and D. Estrin, An energy-efficient MAC protocol for wireless sensor networks. In IEEE INFOCOM, volume 3, pages 1567-1576, June 2002.

[3] W. Ye, J. Heidemann, and D. Estrin, "Medium Access Control With Coordinated Adaptive Sleeping for Wireless Sensor Networks". IEEE/ACM Transactions on Networking, Volume: 12, Issue: 3, Pages: 493 - 506, June 2004.

[4] T. V. Dam and K. Langendoen, “An Adaptive Energy-Efficient MAC Protocol for Wireless Sensor Networks”. SenSys'03, Los Angeles, Pages 171 - 180. Nov 2003.

[5] P. Lin, C. Qiao, and X. Wang, "Medium Access Control With A Dynamic Duty Cycle For Sensor Network". IEEE WCNC'04, March 2004.

[6] LAN MAN Standards Committee of the IEEE Computer Society, "Wireless LAN medium access control (MAC) and physical layer (PHY) specification”. IEEE, New York, NY, USA, IEEE Std 802.11-1997 edition, 1997.

[7] T. Zheng, S. Radhakrishnan, and V. Sarangan, "PMAC: An adaptive energy-efficient MAC protocol for wireless sensor networks," in Proc.IPDPS, 2005.

[8] V. Shnayder, M. Hempstead, B. Chen, G. W. Allen, and M.Welsh, "Simulating the power consumption of large-scale sensor network applications". In SenSys '04 pages 188-200, New York, NY, USA, 2004. ACM Press.

[9] S. Narayanaswamy, V. Kawadia, R. S. Sreenivas, and P. R. Kumar, Power Control in Ad-Hoc Networks: "Theory, Architecture, Algorithm and Implementation of the COMPOW protocol". In European Wireless 2002, February 2002.

[10] G. Simon, P. Volgyesi and A. Ledeczi, "Simulation-based optimization of communication protocols for large-scale wireless sensor networks". IEEE Aerospace, March 2003.

[11] K.T. Kim, H.S. Kim, and H.Y. Youn, "Optimized Clustering for Maximal Lifetime of Wireless Sensor Networks", EUC 2006, LNCS 4097, pp. 465 - 474.

[12] K.T. Kim and H.Y. Youn, "PEACH: Proxy-Enable Adaptive Clustering Hierarchy for Wireless Sensor network", Proceeding of the 2005.

[13] Pei Huang, Li Xiao, Soroor Soltani,Matt W. Mutka, and Ning Xi."The Evolution of MAC Protocols in Wireless Sensor Networks: A Survey” IEEE Communications Surveys \& Tutorials, 2012.

[14] Abdelmalik Bachir, Mischa Dohler, Thomas Watteyne, K. Leung "MAC Essentials for Wireless Sensor Networks", IEEE Communications Surveys \& Tutorials, Vol. 12, No. 2, Second Quarter 2010

[15] Vehbi C. Gungor, P. Hancke,"Industrial Wireless Sensor Networks: Challenges,Design Principles, and Technical Approaches", IEEE Transactions on Industrial Electronics, Vol. 56, No. 10, October 2009.

[16] Demirkol and C. Ersoy, "Energy and delay optimized contention for wireless sensor networks",Computer Networks: The International Journal of Computer and Telecommunications Networking,, Vol. 53,Issue 12, pp. 2106-2119, August 2009.

[17] Khaldoun Al Agha, Marc-Henry Bertin, Tuan Dang,"Which Wireless Technology for Industrial Wireless Sensor networks? The Development of OCARI Technology" IEEE Transactions on Industrial Electronics, Vol. 56, No. 10, October 2009.

[18] Rhee, I., Warrier, A., Aia, M., and Min, J "ZMAC: A hybrid MAC for wireless sensor networks". Proc. Of the 3rd ACM Conference on Embedded Networked Sensor Systems (2005)

\section{AUTHORS}

\section{PANDEESWARAN CHELLIAH}

Graduated from Madurai Kamaraj University with B.E ( Instrumentation and Control). M.Tech (Applied Electronics) from Dr.M.G.R University, Chennai. He has worked as faculty in Electronics and Instrumentation Engg in Jaya Engg College, Chennai for more than 8 years. He is with St.Joseph's College of Engineering, Chennai, Tamil Nadu, India for more than 7 years. He is a part time research scholar in MIT Anna University Chennai. His field of Interests are Microprocessor and Microcontrollers, Embedded systems, Wireless Sensor Networks and Industrial Automation.

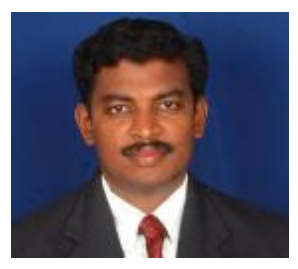


PAPA NATARAJAN

Graduated from Annamalai University with B.E (Electronics and Instrumentation). M.Tech (Digital Electronics) from Cochin University. She has worked as faculty in Instrumentation Engg in Annamalai University for 5 years. She is with Anna University for more than 10 years. She has carried out Ph.D in "Nonlinear control of Heat Exchanger". Her field of interests are process control, Industrial Automation and VLSI Design.

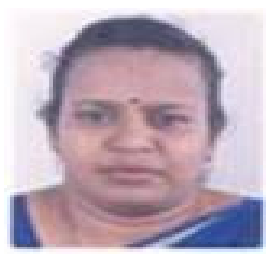

\section{JAYESH SUNDAR GOPINATH}

Graduated from Thangavelu Engineering College(affiliated to Anna University), Chennai, with B.E in Electronics and communication and currently pursuing final year in M.E Control and Instrumentation at St.Joseph's College Of Engineering(affiliated to Anna University), Chennai, Tamil Nadu, India. He has been a part of the research team and presented papers in the field of Industrial wireless sensor network. His field of interest are industrial wireless sensor networks and industrial instrumentation.

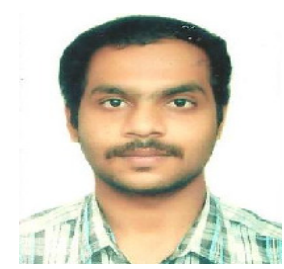

